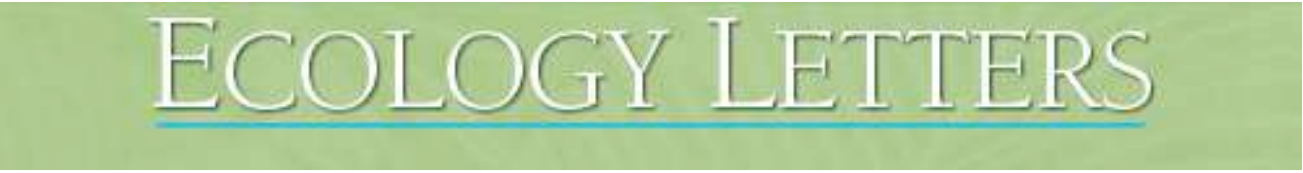

\title{
Antagonistic competition moderates virulence in Bacillus thuringiensis.
}

\begin{tabular}{|r|l|}
\hline Journal: & Ecology Letters \\
\hline Manuscript ID: & ELE-01037-2010.R2 \\
\hline Manuscript Type: & Letters \\
\hline Author: & $\mathrm{n} / \mathrm{a}$ \\
\hline Complete List of Authors: & $\begin{array}{l}\text { Garbutt, Jennie; University of Oxford, Zoology } \\
\text { Bonsall, Michael; University of Oxford, Department of Zoology } \\
\text { Wright, Denis; Imperial College London, Biology } \\
\text { Raymond, Ben; Royal Holloway University of London, Biological } \\
\text { Sciences }\end{array}$ \\
\hline Key Words: & $\begin{array}{l}\text { antagonism, Bacillus thuringiensis, evolution of virulence, } \\
\text { interference competition, mixed infection, social evolution, trade-off }\end{array}$ \\
\hline & \multicolumn{2}{|l}{} \\
\hline
\end{tabular}




\title{
Antagonistic competition moderates virulence in
}

Bacillus thuringiensis.

\author{
Jennie Garbutt ${ }^{1 \dagger}$, Michael B. Bonsall ${ }^{1}$, Denis J. Wright ${ }^{2}$, Ben Raymond ${ }^{1,3 *}$ \\ ${ }^{1}$ Mathematical Ecology Research Group, Dept of Zoology, University of Oxford, \\ South Parks Road, Oxford, OX1 3PS, UK, michael.bonsall@zoo.ox.ac.uk \\ ${ }^{2}$ Division of Biology, Faculty of Natural Science, Imperial College London, Silwood \\ Park campus, Ascot, SL5 7PY, UK, d.wright@imperial.ac.uk \\ ${ }^{3}$ School of Biological Sciences, Royal Holloway University of London, Egham, \\ Surrey, TW20 OEX, UK. \\ *Correspondence: Email: ben.raymond@rhul.ac.uk; tel. 004417843443547 \\ ${ }^{\dagger}$ Current address: Department of Biology and Biochemistry, University of Bath, \\ Claverton Down, Bath, BA2 7AY, UK, jennie.garbutt@gmail.com
}

\author{
Running title: "Antagonism moderates virulence" \\ Words in abstract 147; Words in main text 4948; Words in whole MS 6863; 6 \\ Figures; 0 Tables; 49 References
}

\begin{abstract}
Author contributions: BR, DJW \& MBB conceived experiments; JG performed experiments; BR analysed the data; JG \& BR wrote the first manuscript draft; all authors contributed to revisions.
\end{abstract}




\begin{abstract}
Classical models of the evolution of virulence predict that multiple infections should select for elevated virulence, if increased competitiveness arises from faster growth. However, diverse modes of parasite competition (resource-based, antagonism, immunity manipulation) can lead to adaptations with different implications for virulence. Using an experimental evolution approach we investigated the hypothesis that selection in mixed strain infections will lead to increased antagonism that trades off against investment in virulence. Selection in mixed infections led to improved suppression of competitors in the bacterial insect pathogen Bacillus thuringiensis. Increased antagonism was associated with decreased virulence in three out of four selected lines. Moreover, the presence of a competitor within a host decreased the virulence of co-infections, and tended to decrease pathogen growth in vivo and in vitro. Spiteful interactions among these bacteria may be favoured because of the high metabolic costs of virulence factors and the high risk of mixed infections.
\end{abstract}

\title{
Keywords
}

antagonism, Bacillus thuringiensis, evolution of virulence, interference competition, mixed infection, social evolution, trade-off, 


\section{INTRODUCTION}

Competition between parasites in mixed infections can have diverse implications for virulence (Mideo 2009). If pathogens primarily compete for resources based upon differential replication rates, then mixed infections are predicted to select for increased virulence (Nowak \& May 1994; van Baalen \& Sabelis 1995; Frank 1996; Mosquera \& Adler 1998) and experimental evidence indicates that the competitive outcome between different strains sharing a host can be positively correlated with virulence (de Roode et al. 2005; Bell et al. 2006; Ben-Ami et al. 2008). However, other studies have shown that the relationship between virulence and competitive ability varies considerably (Turner \& Chao 1999; Hodgson et al. 2004; Hughes \& Boomsma 2004; Gower \& Webster 2005; Harrison et al. 2006).

The complexity of real competitive interactions within hosts is much broader than simple resource competition. Kin-selection approaches to parasite competition, for example, have very different predictions for virulence evolution. If virulence is based on the manufacture and export of extra-cellular virulence factors (i.e. public goods), investment in these public goods has a cost that can be manifest decrease in pathogen replication rates (Brown et al. 2002). Social evolutionary theory predicts that low relatedness between strains in a mixed infection may lead to the evolution of cheating genotypes. Cheats will have low virulence in single strain infections but a growth advantage in mixed infections (Turner \& Chao 1999; Brown et al. 2002). Interference competition and the host immune system, with or without host manipulation by parasites, can also have strong effects on competitive outcomes and on virulence 
(Taylor et al. 1998; Davies et al. 2002; Brown et al. 2006; Brown et al. 2009). Adaptations to evade manipulation of the host inflammatory response prompted by competitors, such as the polysaccharide capsule of Streptococcus pneumoniae, can increase the likelihood of virulent infections, even though the meningitis arising from these highly damaging infections cannot lead to effective transmission (Lysenko et al. 2010). The complexity of within-host processes can therefore lead to diverse optimal strategies for coping with competitors, strategies that have distinct implications for virulence evolution as observed in comparisons between hosts or populations of hosts (Mideo 2009; Alizon 2008).

Secreted antagonists are widespread in bacteria, thus interference competition may be particularly common in mixed bacterial infections. Bacteria can produce antibiotics (aimed at taxonomically distant competitors) and highly diffusible peptides, known as bacteriocins, that target taxonomically closer strains (Riley \& Wertz 2002). Investment in behaviour that lowers the fitness of distantly related neighbours is an example of spite, as opposed to cooperation in which individuals invest in behaviours that increase the fitness of related neighbours (Gardner et al. 2004). Bacteriocin genes are closely linked to appropriate resistance genes, so that the carrier and its progeny are immune to the effects of these peptides (Riley \& Wertz 2002). These genes can therefore be classed as spiteful green beards: costly behaviours that reduce the fitness of non-kin (Dawkins 1976).

Despite enormous interest in the effect of mixed infections on the evolution of virulence, most studies are restricted to simple competition experiments that compare overall virulence (the disease induced mortality of a co-infected host) with the 
virulence of singly infected hosts (Alizon 2008). Few studies have directly tested how the selection imposed by mixed infections affects virulence (Turner \& Chao 1999) and none have tested how selection in antagonistic mixed infections will affect virulence. Given the evidence for the social nature of bacteriocins (Massey et al. 2004; Inglis et al. 2009) we tested the theoretical predictions deriving from a kinselection approach to bacterial antagonism (Gardner et al. 2004). In their work Gardner et al. (2004) predicted that investment in spite should peak at intermediate levels of kinship. When genotypes are rare, total production of bacteriocins will tend to be low and ineffectual. Similarly, when genotypes are at high frequencies investment in bacteriocins is less worthwhile as there are fewer targets for antagonism. Increased investment in spite should lead to reduced virulence in single infections, because investment in antagonism is expected to be metabolically costly (Gardner et al. 2004).

In this study we use an experimental evolution approach to test whether selection in mixed versus single strain infections leads to altered investment in spite and changes in pathogen virulence. We use the Gram-positive, spore-forming insect pathogen Bacillus thuringiensis Berliner, and one of its hosts Plutella xylostella, the diamondback moth. Numerous bacteriocins have been described in B. thuringiensis and among its relatives in the Bacillus cereus group (Favret \& Yousten 1989; Cherif et al. 2003). This bacterium is a biological control agent, and is the source of Cry toxins integrated into genetically modified crops for insect control (Glare \& O'Callaghan 2000). These bacteria infect hosts orally, the Cry toxins (which are essential virulence factors) solubilize and create pores in the midgut, enabling haemolymph invasion and replication in the host (Schnepf et al. 1998). The Cry 
toxins are also cooperative: solubilized toxins allow a wide range of coinfecting Bacillus strains to enter the host (Raymond et al. 2007). Avirulent strains, or social cheats, can then outcompete virulent bacteria within the host because the synthesis of Cry toxin imposes a substantial growth disadvantage (Raymond et al. 2007). Since solubilization is essential for Cry toxin activity, pathogens cannot prevent nonpathogenic competitors from exploiting their investment in virulence, other than by producing antagonists.

The evolutionary and parasitology literature is beset by diverse definitions of virulence. Bacillus thuringiensis must kill its host in order to replicate (Raymond et al. 2008), and can be described as an "obligate killer" (Ebert \& Weisser 1997). Here, we define virulence in terms of the timing of host mortality (host death rate). This timing does show important trade-offs as total spore productivity in cadavers peaks at intermediate death rates (Raymond et al. 2009). We used two strains of $B$. thuringiensis $(B t)$ in this study: a rifampicin resistant strain of Bt. subsp. kurstaki (Btk rif $^{\mathrm{R}}$ ) which is pathogenic to $P$. xylostella and a range of Lepidoptera; and a spectinomycin resistant strain of the Bt. biovar. tenebrionis $\left(B t t \operatorname{spec}^{\mathrm{R}}\right) . B t t \operatorname{spec}^{\mathrm{R}}$ is not pathogenic to $P$. xylostella, but is not a social cheat as it expresses Cry toxins that are pathogenic to Coleoptera. The $B t k$ rif $^{\mathrm{R}}$ strain possesses the MLST genotype ST 8, which dominates terrestrial Bt populations in the UK (Raymond et al. 2010). Diverse $B t$ genotypes share the soil as a reservoir and can co-occur on the same leaf in natural populations, making mixed infections highly likely (Collier et al. 2005). This study found that passage in mixed strain infections selected for pathogens better able to suppress the growth of competitors. The evolution of increased competitive ability (antagonism) was associated with reduced virulence. Furthermore, mixed infections 
were always less virulent than single strain infections and tended to reduce pathogen density.

\section{MATERIAL AND METHODS}

\section{Bacterial and insect strains}

The pathogenic strain Btk rif $^{\mathrm{R}}$ was isolated from the commercial biopesticide preparation, DiPel WP (Valent Biosciences) (Raymond et al., 2007); Btt $\operatorname{spec}^{\mathrm{R}}$ was provided by DJI Thomas (HRI, Wellesbourne, UK). The difference in resistance to the two antibiotics enables the selective isolation of each strain from insects infected with both genotypes. A Btk susceptible population of diamondback moth, $P$. xylostella (supplied by Exosect Ltd, Southampton, UK) was maintained at $25^{\circ} \mathrm{C}$ on 4 5 week old greenhouse-grown Chinese cabbage, Brassica pekinensis cultivar "One Kilo S.B.”.

\section{Selection experiment}

We selected for improved reproduction of $B t k$ rif $^{\mathrm{R}}$ in live hosts with a passage protocol using four independent lineages per experimental treatment. We imposed a single strain selection treatment (SS selection) and a mixed strain selection treatment (MS selection). In the SS treatment we used leaf inocula of 2000 spores $\mu \mathrm{l}^{-1}$ of Btk rif $^{\mathrm{R}}$; in the MS treatment we used 2000 spores $\mu \mathrm{l}^{-1} B t k \operatorname{rif}^{\mathrm{R}}$ and 500 spores $\mu \mathrm{l}^{-1}$ of Btt $\operatorname{spec}^{\mathrm{R}}$. This maintained a fixed dose of the pathogenic strain and ensured that mortality rates in the two treatments were very similar at the beginning of the passage experiment (Fig. 1). The Btt $\operatorname{spec}^{\mathrm{R}}$ strains were allowed to coevolve with $B t k$ rif $^{\mathrm{R}}$ in the MS treatment. Leaves were inoculated with controlled doses at each passage as per standard leaf dip procedures, which deposit approximately $200 \mu 1$ of inocula on 
each leaf (Raymond et al. 2005). Five third instar diamondback moth larvae were then confined on individual leaf discs for seven days in 50mm Petri dishes. Selection procedures are described in Fig. 1. Cadavers from each Petri dish were pooled and homogenized together in $500 \mu \mathrm{l}$ saline in a single microcentrifuge tube with a pellet pestle, and plated onto Bacillus cereus specific agar (BcSA, Oxoid) containing selective antibiotics (Fig. 1a). This pooling facilitates global competition between cadavers: insects containing higher densities of pathogens contribute more progeny to this pool and are better represented in the next round of selection (Griffin et al. 2004). We also selected for bacterial density between cadaver pools isolated from each leaf disc (there being 10 leaf discs for each replicate lineage, Fig. 1a) only the five cadaver pools with the highest population densities contributed spores to the next round of selection (Fig. 1a).

The selection experiment was continued until passage four, at which point the change in competitive ability of $B t k$ rif $^{\mathrm{R}}$ was such that no $B t t \operatorname{spec}^{\mathrm{R}}$ colonies could be isolated from cadavers in the mixed infection treatment. Significant difference in the mortality rates between bacteria in the MS and SS treatments emerged at passage four (Fig. 1b) (using strains isolated from the third passage) and strains isolated after the third passage were used in all experiments in this study.

\section{Qualitative assays for secreted antagonists in Btk rif $^{\mathrm{R}}$}

Antagonistic competition in bacteria tends to be mediated by diffusible molecules that inhibit the growth of competitors. These soft-agar overlay assays assessed whether $B t k$ rif $^{\mathrm{R}}$ colonies secrete diffusible antagonistic compounds capable of inhibiting the growth of $B t t \operatorname{spec}^{\mathrm{R}}$ on solid aritificial media. Colonies were initiated with $5 \times 10^{6}$ spores of $B t k$ rif $^{\mathrm{R}}$ in $10 \mu$ of saline $(0.85 \% \mathrm{NaCl})$ on $90 \mathrm{~mm}$ hard agar LB plates $(1.2 \%$ 
bacteriological agar, Oxoid) and left to grow overnight at $30^{\circ} \mathrm{C}$. Bacterial colonies were then killed with chloroform-soaked filter paper; this prevents the experimental strain from contaminating the tester culture but does not destroy the bacteriocins. Tester cultures were prepared by 1000-fold dilutions of overnight cultures of $B t t \operatorname{spec}^{\mathrm{R}}$ $\left(5 \mathrm{ml} \mathrm{LB}\right.$ broth, $\left.37^{\circ} \mathrm{C}, 180 \mathrm{rpm}\right)$ in soft agar $\left(\mathrm{LB}\right.$ with $0.6 \%$ agar at $\left.43^{\circ} \mathrm{C}\right)$. Residual chloroform was allowed to evaporate for at least 5 minutes before $4 \mathrm{ml}$ of soft agar containing the tester culture was poured over each plate. Plates were then incubated overnight before being visually assessed for inhibition of the tester cultures. These assays used SS and MS lineages $(\mathrm{N}=8)$ as well as $B t t \operatorname{spec}^{\mathrm{R}}$ controls; each isolate was replicated twice.

\section{Quantitative in vitro competition assays}

In these competition experiments the eight passaged $B t k$ rif $^{\mathrm{R}}$ strains were cultured on their own or with the ancestral $B t t \operatorname{spec}^{\mathrm{R}}$ strain on solid artificial media in order to investigate how the presence of a competitor affected bacterial growth and whether increased competitive ability was associated with a fitness cost. Competition experiments were initiated with known densities and ratios of $B t k \operatorname{rif}^{\mathrm{R}}$ and $B t t \operatorname{spec}^{\mathrm{R}}$ strains. Experiments used LB broth grown spores (heat treated to $65^{\circ} \mathrm{C}$ for 30 minutes to ensure physiological uniformity of cultures). Competition experiments used $1.5 \%$ agar LB plates $(90 \mathrm{~mm})$ containing $0.5 \mathrm{mM}$ ferric chloride, and experiments were initiated with approximately 150 colonies per plate of both Btk and Btt (three plates per treatment). Assay plates were cultured for 5 days at $30^{\circ} \mathrm{C}$ after which bacterial cells were recovered by rolling sterile glass beads over the plate surface in $5 \mathrm{ml}$ saline. Initial densities were calculated from the number of colonies appearing on plates in the first 24 hours; final densities of $B t k$ rif $^{\mathrm{R}}$ and $B t t \operatorname{spec}^{\mathrm{R}}$ were determined by dilution 
plating of recovered biomass. Ratios of competitors were measured by streaking individual colonies (from plates of diluted mixtures) onto media with selective antibiotics. The log ratio of the change in density (in colony forming units) was used to calculate relative growth rate $\left(\mathrm{RGR}=\log _{\mathrm{e}}\right.$ [final density/initial density] $)$ and the final total density on plates was used as measure of bacterial productivity. Relative fitness was calculated as the ratio of RGRs (RGR $B t k \mathrm{rif}^{\mathrm{R}} / \mathrm{RGR} B t t \mathrm{spec}^{\mathrm{R}}$ ).

\section{Bioassays of pathogen virulence}

The ancestral $B t k$ rif $^{\mathrm{R}}$ strain, the eight passaged $B t k$ rif $^{\mathrm{R}}$ strains and the ancestral Btt $\operatorname{spec}^{\mathrm{R}}$ strain were grown on LB agar plates for 5 days at $30^{\circ} \mathrm{C}$, after which biomass was recovered, washed three times in saline and pasteurized by heating to $65^{\circ} \mathrm{C}$ for 30 minutes. Cell density was measured with a haemocytometer and suspensions were diluted in saline with $100 \mu \mathrm{g} \mathrm{ml}^{-1}$ triton-X-100. Chinese cabbage leaf discs (7 per treatment) were inoculated via standard leaf-dip procedures and larvae infected by feeding as in the passage experiment above (Raymond et al. 2005). Two experiments were conducted simultaneously: the first tested the effects of co-infection on the virulence of all Btk rif $^{\mathrm{R}}$ strains: insects were infected with each ancestral and selected $B t k$ rif $^{\mathrm{R}}$ strains alone (at 250 spores $\mu \mathrm{l}^{-1}$ ) or with ancestral $B t t \operatorname{spec}^{\mathrm{R}}$ (also 250 spores $\mu l^{-1}$ ) in a mixed infection. In the second experiment bioassays of ancestral and selected $B t k$ rif $^{\mathrm{R}}$ strains were made using three doses $\left(250\right.$ spores $\mu \mathrm{l}^{-1}, 500$ spores $\mu \mathrm{l}^{-1}$ and 1000 spores $\mu^{-1}$ ) and an uninfected control. All bioassays used 35 larvae per dose. Insects were scored for mortality daily for five days, at which point survivors are either pupae or fourth-instar larvae that are unlikely to succumb to infection. Spores were collected from cadavers using the methods described above; cadavers collected from a single leaf disc were treated as a single replicate. 


\section{Statistical analysis}

Data analysis was carried out in R v 2.6 .2 (www.r-project.org/) using mixed-effects models that incorporated independent evolutionary lineages as random effects. The significance testing of fixed factors used the sequential removal of model terms following fitting of maximum likelihood models. Survivorship analysis used Cox proportional hazard models, nesting of strains within selection treatments was accounted for using the term "cluster" which allows for the correlation of observations within strain (Tableman \& Sung Kim 2004). Model simplification in survivorship analysis was conducted using likelihood ratio tests and comparisons of AIC (Tableman \& Sung Kim 2004). Standard errors for survival data were calculated by fitting Kaplan Meier survivorship curves using the default survfit function (Tsiatis 1981). Model assumptions were checked with graphical analyses of error distribution and normality or with explicit tests of the assumption of constant hazard (function cox.zph).

\section{RESULTS}

\section{Effect of selection treatment on competition between $B t t$ and $B t k$}

Qualitative assays confirmed that the ancestral and selected Btk rif $^{\mathrm{R}}$ strains could secrete a diffusible antagonist capable of suppressing the growth of the ancestral Btt $\operatorname{spec}^{\mathrm{R}}$ strain: the assays showed that 1 day old colonies produced a clear zone of inhibition approximately $2 \mathrm{~mm}$ in radius. Control assays using $B t t \operatorname{spec}^{\mathrm{R}}$ found no evidence of self-inhibition. These qualitative assays did not, however, reveal any obvious difference in the strength of inhibition among the $B t k$ rif $^{\mathrm{R}}$ strains from the different selection treatments. 
However, quantitative in vitro competition assays on agar plates allowed us to measure the growth rates of $B t k$ rif $^{\mathrm{R}}$ lineages from the two selection treatments both alone and in competition with ancestral $B t t \operatorname{spec}^{\mathrm{R}}$. The common garden competition experiment showed that $B t k$ rif $^{\mathrm{R}}$ lineages from the mixed strain selection regime (MS lineages) were better at suppressing the growth of $B t t \operatorname{spec}^{\mathrm{R}}$ than $B t k$ rif $^{\mathrm{R}}$ lineages from the single strain selection treatment (SS lineages) (Fig. 2; mixed model ANOVA of relative growth rate $\sim$ selection treatment [fixed factor], random $=\sim 1$ lstrain/plate; likelihood ratio $=8.11$, d.f. $=1, P=0.0044)$. MS lineages had higher relative fitness when grown in competition with $B t t \operatorname{spec}^{\mathrm{R}}$ (Fig. 2; mixed model ANOVA; likelihood ratio $=6.12$, d.f. $=1, P=0.0134)$. The final density of $B t t \operatorname{spec}^{\mathrm{R}}$ was also lower when in competition with MS lineages (Fig. 3 [Btt only]; mixed model ANOVA; likelihood ratio $=6.13$, d.f. $=1, P=0.0133$ ). Finally, if resource competition was mediating interactions between these strains we would expect the growth of one strain to be negatively correlated with that of its competitor, we found the opposite pattern in comparisons between plates (but within strains): $B t t \operatorname{spec}^{\mathrm{R}}$ reproduction in vitro was positively correlated with $B t k$ rif $^{\mathrm{R}}$ reproduction (mixed model ANOVA; likelihood ratio $=11.00$, d.f. $=1, P<0.0001$, data not shown).

We also used these in vitro competition experiments to investigate (a) whether increased investment in antagonism had a measurable pleiotropic effect on fitness (of strains grown in isolation) and (b) whether competition with ancestral $B t t \operatorname{spec}^{\mathrm{R}}$ was costly at the population level, with the expectation that antagonism should reduce the efficiency of resource utilization and therefore final bacterial densities. The evidence for a pleiotropic fitness cost was equivocal for strains grown in isolation: bacterial productivity (final densities) tended to be lower for MS lineages (Fig. 3 [Btk control]; 
mixed model ANOVA; likelihood ratio $=3.79$, d.f. $=1, P=0.052$ ). There was no difference in RGR between SS and MS lineages (mixed model ANOVA; likelihood ratio $=1.43$, d.f. $=1, P=0.32$, data not shown), although measurements of RGR are subject to additional stochastic variation in the initial density of colonies on plates. However, when analysing all the data on $B t k$ rif $^{\mathrm{R}}$ productivity MS lineages had lower productivity than SS lineages (Fig. 3 [Btk control \& Btk in competition]; mixed model ANOVA; likelihood ratio $=8.54$, d.f. $=1, P=0.0035)$. In addition, competition itself was clearly costly and antagonistic, as the presence of the $B t t \operatorname{spec}^{\mathrm{R}}$ competitor decreased total bacterial productivity (Fig. 3 [Btk+Btt \& Btk control], mixed model ANOVA: effect of $B t t$ addition, likelihood ratio $=19.3$, d.f. $=1, P<$ 0.001) and this decrease was greatest for the MS lineages (Fig. 3 [Btk+Btt \& Btk control], selection treatment $* B t t$ addition interaction- likelihood ratio $=9.74$, d.f. $=1$, $P=0.0018)$

\section{Bioassays and in vivo competition assays}

We used a nested Cox proportional hazard survivorship analysis to test two hypotheses: (1) that MS lineages had lower virulence (or rate of death) in single strain infections than both their ancestor and SS lineages; and (2) that coinfection (addition of the ancestral $B t t \operatorname{spec}^{\mathrm{R}}$ competitor) decreased overall virulence relative to single strain infections. The full statistical model was: coxph (Surv(death,status) cluster(strain $)+$ selection treatment $*$ as.factor(dose $)+B t$ addition; where selection treatment, dose and effect of $B t t \operatorname{spec}^{\mathrm{R}}$ are fixed factors. This analysis supported both hypotheses. There was a strong effect of selection treatment on mortality rate (main effect of selection treatment: likelihood ratio test- d.f. $\left.=2, \chi^{2}=119, P<0.0001\right)$. SS lineages had increased virulence relative to the MS lineages after accounting for the 
effect of coinfection (robust $\mathrm{z}$ test: hazard coefficient $0.7776, \mathrm{z}=2.48, P=0.013$; Fig. 4). There was also a significant interaction between bioassay dose and selection treatment (likelihood ratio test, d.f. $=4, \chi^{2}=12.53, P=0.014$ ). At the lowest dose, MS lineages showed decreased virulence relative to both SS lineages (robust $\mathrm{z}$ test: hazard coeff. $-0.931, \mathrm{z}=-3.09, P=0.002$ ) and the ancestral strain (robust $\mathrm{z}$ test: hazard coeff. $-0.871, \mathrm{z}=-2.93, P=0.003$ ) (see Fig. S1 in Supporting Information). At the two highest doses the difference between the MS lineages and the ancestral strain was less marked (dose 500* treatment interaction, hazard coeff. $=-0.632, \mathrm{z}=$ 9.28, $P<0.0001 ;$ dose $1000 *$ treatment interaction, hazard coeff. $=-0.818, \mathrm{z}=-3.32$, $P=0.0009$ ) (Fig. S1). No larval mortality was observed in the uninfected control treatment.

There was heterogeneity in the virulence of the MS lineages. Analyses using individual lineages (rather than selection treatment) as explanatory variables demonstrated that three out of the four MS lineages had reduced virulence relative to the ancestral Btk rif $^{\mathrm{R}}$ strain (Appendix S1, Fig. 4), while lineage R3KT had both high virulence and high relative fitness in the presence of $B t t \operatorname{spec}^{R}$ (Fig. 2). However, if we discounted the outlier (lineage R3KT) we could discern a significant negative correlation between virulence (i.e. predicted hazard coefficients) and the relative fitness of strains in competition with $B t t \operatorname{spec}^{\mathrm{R}}\left(F_{1,5}=7.94, P=0.037\right.$, Fig. S2). Strain-level analyses of time to kill (the time to death of all insects with successful infections) showed qualitatively similar patterns to survivorship analyses (Appendix S1).

The presence of $B t t \operatorname{spec}^{\mathrm{R}}$ in co-infections reduced the overall virulence of the pathogenic Btk rif $^{\mathrm{R}}$ in survivorship analyses (Fig. 5, robust $\mathrm{z}$ test; hazard coeff. = 0.36, $z=-2.4, P=0.017)$. Although there was between-strain variation in the 
response of mortality rates to coinfection (Fig. 5) there was no difference in the effect of $B t t \operatorname{spec}^{\mathrm{R}}$ on MS and SS lineages (Fig. 5, robust z test; hazard coeff $=0.05, z=-$ 0.18, $P=0.86)$. Analyses of time to kill found no significant effect of $B t t \operatorname{spec}^{\mathrm{R}}$ addition (Appendix S1), suggesting that coinfection affected virulence primarily through altering total proportional mortality rather than the rate at which infections killed hosts (Fig. 5). In addition, we tested how coinfection with $B t t \operatorname{spec}^{\mathrm{R}}$ affected the abundance of Btk rif $^{\mathrm{R}}$ spores within hosts, with the expectation that the reduction in overall virulence would be associated with reduced replication in the pathogenic strain. The effect of coinfection on $B t k$ rif $^{\mathrm{R}}$ reproduction was marginally nonsignificant (mixed model ANOVA: log counts selection treatment * Btt addition, random $=\sim 1$ Strain, with selection treatment and Btt addition as fixed effects, Likelihood ratio $=3.79, P=0.051$, Fig. $6 a)$. There was no significant interaction between coinfection and selection treatment (mixed model ANOVA, Likelihood ratio $=1.01, P=0.315)$ and no effect of selection treatment on the number of spores recovered from hosts (Likelihood ratio $=0.331, P=0.365$ ).

We expected increased antagonism to lead to some in vivo reproductive benefit for the MS lineages in the presence of their competitor. We compared relative fitness based upon the change in proportion of the $B t t \operatorname{spec}^{\mathrm{R}}$ strains in cadavers relative to initial inoculum: $x_{2}\left(1-x_{1}\right) / x_{1}\left(1-x_{2}\right)$, where $x_{1}$ is the initial proportion of $B t t \operatorname{spec}^{\mathrm{R}}$ and $x_{2}$ is the observed final proportion in cadavers. While the data showed a trend in the expected direction (Fig. 6a), there were no significant differences in relative fitness between MS and SS lineages (mixed effect ANOVA, passage treatment as fixed effect, strain as random effect, Likelihood ratio $=2.19, P=0.14$ ).

One explanation for the absence of a significant effect is that within-cadaver pathogen densities in this competition experiment were too low for $B t k$ rif $^{\mathrm{R}}$ to reap a 
reward from investment in antagonism. We therefore examined the relationship between the density of $B t t \operatorname{spec}^{\mathrm{R}}$ and $B t k \operatorname{rif}^{\mathrm{R}}$ for MS and SS lineages while competing within cadavers. There was a very strong interaction between the slope of this relationship and passage treatment (Fig. 6b; mixed model ANOVA: log Btt counts $\log B t k$ counts $*$ selection treatment, random $=\sim 1 \mid$ Strain, fixed factors are $\log B t k$ counts and treatment, d.f. $=1$, likelihood ratio $=61.6, P<0.0001)$. Growth of Btt $\operatorname{spec}^{\mathrm{R}}$ and Btk rif $^{\mathrm{R}}$ was positively correlated within hosts for SS lineages but uncorrelated for MS lineages (post hoc test for non-zero slope, coeff $==-.0005, t=$ 0.53, $P=0.99$ ). Speculatively, this suggests that when MS lineages were at high density within hosts, $B t t \operatorname{spec}^{\mathrm{R}}$ competitors were held at low density.

\section{DISCUSSION}

Through several independent experiments we have shown that the $B t$ strains (Btt $\operatorname{spec}^{\mathrm{R}}$ and $B t k$ rif $^{\mathrm{R}}$ ) can interact antagonistically, potentially via a diffusible molecule. We found reduced bacterial densities and virulence in mixed strain infections, a pattern also shown under bacteriocin-mediated antagonism in other bacterial species in insect hosts (Massey et al. 2004; Inglis et al. 2009). The presence of a competitor in vitro also reduced total bacterial productivity. Neither this work, nor previous studies found a negative correlation between growth rates of the two competing pathogens between hosts (Raymond et al. 2007), as would be expected under strict resource-based competition. The differences in evolved lineages from single and mixed infection selection regimes indicate that mixed infection selected for increased antagonism. All four lineages of $B t k$ rif $^{\mathrm{R}}$ from the mixed infection selection regime evolved an improved ability to suppress the growth of $B t t \operatorname{spec}^{\mathrm{R}}$ in competition assays. Elevated investment in antagonism is expected to be costly and improved 
suppression of competitors in vitro incurred a cost in terms of final density. Our interpretation of these results is that the mixed strain selection regime led to the evolution of increased levels of antagonism in $B t k$ rif $^{\mathrm{R}}$.

One anticipated result was lacking from these experiments: we expected increased investment in antagonism to yield a pay-off in the presence of the Btt $\operatorname{spec}^{\mathrm{R}}$ competitor. The MS lineages tended to have a higher relative fitness (in comparison to Btt $\operatorname{spec}^{\mathrm{R}}$ ) in vitro and in vivo. Given that we were selecting for increased productivity in cadavers, we expected that increased investment in antagonism should be rewarded with improved productivity. In contrast, we found that the strains with less antagonism grew as well or better when competing with $B t t \operatorname{spec}^{\mathrm{R}}$ in vivo and in vitro (Fig. 2, Fig. 4). There are two possible explanations. The first is that the benefits of antagonism are density dependent; Fig. 6 suggest that the more antagonistic strains are effective at preventing this competitor reaching a high density but that only a small proportion of infections achieved high densities in our trial. The second, nonmutually exclusive explanation is that the MS lineages are generally less fit. The Btk $\operatorname{rif}^{\mathrm{R}}$ strains originate from a generalist genotype and may be under selection to adapt to the experimental host; the rifampicin resistance marker carries a fitness cost and all strains would be under selection for compensating mutations (Reynolds 2000). The competitor reduces the density of the $B t k$ rif ${ }^{\mathrm{R}}$ strains in the host and will therefore reduce the number of generations of selection experienced by the mixed passage strains. From our observations of density during host passage we estimate that the mixed passage lineages experienced on average 21.4 generations $(\mathrm{se}=2.7)$ in three passages, while the single strain lineages experienced 30.3 generations of selection (se $=1.9)$.

Van Baalen \& Sabelis (1995) noted that the nature of mixed infection 
(coinfection or superinfection) was associated with differences in the type of competition between pathogens or parasites and could in turn determine the theoretical predictions for the evolution of virulence. Given the evidence for antagonistic competition in this study, intra-specific models of coinfection or superinfection will probably poorly approximate the interaction between $B t$ strains in an insect host. We found that increased suppression of a competitor was typically associated with a reduction in virulence, in agreement with our initial hypothesis (Gardner et al. 2004). One simple explanation for this pattern is that investment in antagonistic competition is costly and that increased antagonism is traded-off against either growth in the host or the production of virulence factors (Inglis et al. 2009). Production of colicin-based bacteriocins in Escherichia coli is associated with the very high costs of lethal cell lysis (Chao \& Levin 1981). Production of bacteriocins in gram-positive bacteria is often constitutive, and may be less costly than colicin but still involves the extracellular export of peptides, a metabolically expensive process (Dykes \& Hastings 1997).

An alternative explanation for the evolution of reduced virulence in mixed strain infections is based on the predictions of the evolutionary theory for cooperative public goods. It is clear that since one strain produces virulent toxin $\left(B t k\right.$ rif $\left.^{\mathrm{R}}\right)$ and one strain produces a different suite of toxins $\left(B t t \operatorname{spec}^{\mathrm{R}}\right)$ that relatedness at the locus of $B t$ cry toxin production is reduced in mixed infections. Preliminary data have confirmed that $B t$ cry toxins behave as obligate public goods (B. Raymond unpubl. dat.). Evolutionary modeling of this kind of public good predicts that cooperators will evolve lower levels of investment in traits for which there is reduced relatedness (Brown et al. 2002). While this prediction would explain the increased competitiveness and reduced virulence of the mixed passage strain, it does not explain 
why competitor suppression appeared to be associated with a fitness cost. Decreased investment in host-specific public goods (i.e. the export of costly protein) should lead to increased resources available for growth in broth when these traits are not essential (West \& Buckling 2003), the opposite of the pattern we found. While the effects of relatedness at toxin loci may have had only a small influence on the results of this study it is likely that investment in antagonism public goods are intimately linked in many bacterial pathogens. Cooperative behaviours should be favoured if they could be directed non-randomly at relatives via mechanisms such as kin recognition (Hamilton 1964). Bacteriocins, which can be highly diverse within bacterial species, can be thought of as a spiteful means of altering the mean relatedness in the local environment (Hamilton 1970; Gardner et al. 2004). Given the high cost of Bt toxin genes and the high likelihood of mixed infections with non-pathogenic strains (Collier et al. 2005) it should not be surprising that bacteriocin genes have been found to be closely linked to toxin genes in one strain of Bt (Berry et al. 2002). The high prevalence of costly bacterial exo-products involved in virulence (Smith 2001; West \& Buckling 2003; West et al. 2006), products that are highly likely to be public goods, may therefore go some way in explaining why so many pathogenic bacteria invest in antagonism: they are looking after their own.

\section{ACKNOWLEDGMENTS}

This work was supported by BBSRC, NERC and the Royal Society. We thank Mani Narayanan and Dexter Davis for help with passaging Bt strains. We thank two anonymous reviewers, Samuel Alizon, Andy Gardner and Sam Brown for comments on earlier versions of this paper. 


\section{REFERENCES}

Alizon S. (2008). Decreased overall virulence in coinfected hosts leads to the persistence of virulent parasites. Am. Nat., 172, E67-E79.

Bell A.S., De Roode J.C., Sim D. \& Read A.F. (2006). Within-host competition in genetically diverse malaria infections: parasite virulence and competitive success. Evolution, 60, 1358-1371.

Ben-Ami F., Mouton L. \& Ebert D. (2008). The effects of multiple infections on the expression and evolution of virulence in a Daphnia-endoparasite system. Evolution, 62, 1700-1711.

Berry C., O'Neil S., Ben-Dov E., Jones A.F., Murphy L., Quail M.A., Holden M.T.G., Harris D., Zaritsky A. \& Parkhill J. (2002). Complete sequence and organization of pBtoxis the toxin-coding plasmid of Bacillus thuringiensis subsp. israelensis. Appl. Env. Microbiol., 68, 5082-5095.

Brown S., Le Chat L., De Paepe M. \& Taddei F. (2006). Ecology of microbial invasions: amplification allows virus carriers to invade more rapidly when rare. Curr. Biology, 16, 2048-2052.

Brown S.P., Hochberg M.E. \& Grenfell B.T. (2002). Does multiple infection select for raised virulence? Trends Microbiol., 10, 401-405.

Brown S.P., Inglis R.F. \& Taddei F. (2009). Evolutionary ecology of microbial wars: within-host competition and (incidental) virulence. Evol. Appl., 2, 32-39.

Chao L. \& Levin B.R. (1981). Structured habitats and the evolution of anticompetitor toxins in bacteria. Proc. Natl Acad. Sci. USA, 78, 6324-6328.

Cherif A., Chehimi S., Limem F., Hansen B.M., Hendriksen N.B., Daffonchio D. \& Boudabous A. (2003). Detection and characterization of the novel bacteriocin entomocin 9, and safety evaluation of its producer, Bacillus thuringiensis ssp 
entomocidus HD9. J. Appl. Microbiol., 95, 990-1000.

Collier F.A., Elliot S.L. \& Ellis R.J. (2005). Spatial variation in Bacillus thuringiensis /cereus populations within the phyllosphere of broad-leaved dock (Rumex obtusifolius) and surrounding habitats. FEMS Microb. Ecol., 54, 417-425.

Davies C.M., Fairbrother E. \& Webster J.P. (2002). Mixed strain schistosome infections of snails and the evolution of parasite virulence. Parasitology, 124, $31-38$.

Dawkins R. (1976). The Selfish Gene. Oxford University Press, Oxford.

de Roode J.C., Pansini R., Cheesman S.J., Helinski M.E.H., Huijben S., Wargo A.R., Bell A.S., Chan B.H.K., Walliker D. \& Read A.F. (2005). Virulence and competitive ability in genetically diverse malaria infections. Proc. Natl. Acad. Sci. U. S. A., 102, 7624-7628.

Dykes G.A. \& Hastings J.W. (1997). Selection and fitness in bacteriocin-producing bacteria. Proc. R. Soc. Lond. B., 264, 683-687.

Ebert D. \& Weisser W.W. (1997). Optimal killing for obligate killers: The evolution of life histories and virulence of semelparous parasites. Proc. R. Soc. Lond. B, 264, 985-991.

Favret M.E. \& Yousten A.A. (1989). Thuricin: the bacteriocin produced by Bacillus thuringiensis. J. Invertebr. Pathol., 53, 206-216.

Frank S.A. (1996). Models of parasite virulence. Quart. Rev. Biol., 71, 37-78.

Gardner A., West S.A. \& Buckling A. (2004). Bacteriocins, spite and virulence. Proc. R. Soc. Lond. B, 271, 1529-1535.

Glare T.R. \& O'Callaghan M. (2000). Bacillus thuringiensis: biology, ecology and safety. John Wiley, Chicester.

Gower C.M. \& Webster J.P. (2005). Intraspecific competition and the evolution of 
virulence in a parasitic trematode. Evolution, 59, 544-553.

Griffin A.S., West S.A. \& Buckling A. (2004). Cooperation and competition in pathogenic bacteria. Nature, 430, 1024-1027.

Hamilton W.D. (1964). The genetical evolution of social behaviour. I. J. Theor. Biol., 7, 1-16.

Hamilton W.D. (1970). Selfish and spiteful behaviour in an evolutionary model. Nature, 228, 1218-\&.

Harrison F., Browning L.E., Vos M. \& Buckling A. (2006). Cooperation and virulence in acute Pseudomonas aeruginosa infections. BMC Biol., 4.

Hodgson D.J., Hitchman R.B., Vanbergen A.J., Hails R.S., Possee R.D. \& Cory J.S. (2004). Host ecology determines the relative fitness of virus genotypes in mixed-genotype nucleopolyhedrovirus infections. J. Evol. Biol., 17, 10181025.

Hughes W.O.H. \& Boomsma J.J. (2004). Let your enemy do the work: within-host interactions between two fungal parasites of leaf-cutting ants. Proc. R. Soc. Lond. B, 271, S104-S106.

Inglis R.F., Gardner A., Cornelis P. \& Buckling A. (2009). Spite and virulence in the bacterium Pseudomonas aeruginosa. Proc. Natl Acad. Sci. USA, 106, 5703-7.

Lysenko E.S., Lijek R.S., Brown S.P. \& Weiser J.N. (2010). Within-host competition drives selection for the capsule virulence determinant of Streptococcus pneumoniae. Curr Biol, 20, 1222-6.

Massey R.C., Buckling A. \& Ffrench-Constant R. (2004). Interference competition and parasite virulence. Proc. R. Soc. Lond. Ser. B-Biol. Sci., 271, 785-788.

Mideo N. (2009). Parasite adaptations to within-host competition. Trends Parasitol., $25,261-268$. 
Mosquera J. \& Adler F.R. (1998). Evolution of virulence: a unified framework for coinfection and superinfection. J. Theor. Biol., 195, 293-313.

Nowak M.A. \& May R.M. (1994). Superinfection and the evolution of parasite virulence. Proc. R. Soc. Lond. Ser. B-Biol. Sci., 255, 81-89.

Raymond B., Davis D. \& Bonsall M.B. (2007). Competition and reproduction in mixed infections of pathogenic and non-pathogenic Bacillus spp. J. Invertebr. Pathol., 96, 151-155.

Raymond B., Elliot S.L. \& Ellis R.J. (2008). Quantifying the reproduction of Bacillus thuringiensis HD-1 in cadavers and live larvae of Plutella xylostella. J. Invert. Pathol., 98, 307-313.

Raymond B., Ellis R.J. \& Bonsall M.B. (2009). Moderation of pathogen-induced mortality: the role of density in Bacillus thuringiensis virulence Biol. Letts, 5, 218-220.

Raymond B., Sayyed A.H. \& Wright D.J. (2005). Genes and environment interact to determine the fitness costs of resistance to Bacillus thuringiensis. Proc. $R$. Soc. B., 272, 1519-1524.

Raymond B., Wyres K.L., Sheppard S.K., Ellis R.J. \& Bonsall M.B. (2010). Environmental factors determining the epidemiology and population genetic structure of the Bacillus cereus group in the field. PLoS Path, 6, e1000905. Reynolds M.G. (2000). Compensatory evolution in rifampin-resistant Escherichia coli. Genetics, 156, 1471-81.

Riley M.A. \& Wertz J.E. (2002). Bacteriocins: evolution, ecology, and application. Ann. Rev. Microbiol., 56, 117-137.

Schnepf E., Crickmore N., Van Rie J., Lereclus D., Baum J., Feitelson J., Zeigler D.R. \& Dean D.H. (1998). Bacillus thuringiensis and its pesticidal crystal 
proteins. Microbiol. Mol. Biol. Rev., 62, 775-806.

Smith J. (2001). The social evolution of bacterial pathogenesis. Proc R. Soc Lond. B., $268,61-69$.

Tableman M. \& Sung Kim J. (2004). Survival analysis using S. Chapman \& Hall/CRC, London.

Taylor L.H., Mackinnon M.J. \& Read A.F. (1998). Virulence of mixed-clone and single-clone infections of the rodent malaria Plasmodium chabaudi. Evolution, $52,583-591$.

Tsiatis A. (1981). A large sample study of the estimate for the integrated hazard function in Cox's regression model for survival data. Ann. Statistics, 9, 93-108.

Turner P.E. \& Chao L. (1999). Prisoner's dilemma in an RNA virus. Nature, 398, 441-443.

van Baalen M. \& Sabelis M.W. (1995). The dynamics of multiple infection and the evolution of virulence. Am. Nat., 146, 881-910.

West S.A. \& Buckling A. (2003). Cooperation, virulence and siderophore production in bacterial parasites. Proc. R. Soc. Lond. B, 270, 37-44.

West S.A., Griffin A.S., Gardner A. \& Diggle S.P. (2006). Social evolution theory for microorganisms. Nat. Rev. Microbiol., 4, 597-607.

\section{SUPPORTING INFORMATION}

Additional Supporting Information may be found in the online version of this article:

Appendix S1 Supplementary statistical analyses investigating lineage level heterogeneity in survivorship and time to death in virulence bioassays 
Figure S1 Dose effects on larval mortality imposed by ancestral Btk rif ${ }^{R}$, mixed strain lineages and single strain lineages.

Figure $\mathbf{S 2}$ The relationship between virulence and competitive ability (relative fitness in the presence of $\mathrm{Btt}$ spec $^{R}$ ) of evolved $\mathrm{Btk}$ rif $f^{R}$ lineages 
Figure 1 The passage experiment was set up with four independent lineages in each of the single strain and mixed strain selection treatments (a) represents the experimental protocol for one lineage. At each passage a lineage consisted of ten leaf discs that were used to infect five larvae. Selection was imposed by pooling cadavers isolated from each leaf disc (see text for details) and by propagating bacteria from the five cadaver pools with the highest density. Bacteria for the next passage were propagated from dense areas of bacterial growth on plates, re-plated once to remove potential contaminants and stored as glycerol stocks. Both $B t k$ rif $^{\mathrm{R}}$ and $B t t \operatorname{spec}^{\mathrm{R}}$ strains were passaged and selected in the same manner. (b) Doses were chosen in order to impose similar levels of mortality between treatments at the beginning of the experiment; by passage four significant differences in mortality had emerged between selection treatments (d.f. $\left.=1,6 ; \chi^{2}=10.2, P=0.0018\right)$.

Figure 2 The effect of selection regime (mixed strain selection, single strain selection) on competition between ancestral $B t t \operatorname{spec}^{\mathrm{R}}$ and $B t k \operatorname{rif}^{\mathrm{R}}$ on artificial media. Replicates R1-R4 are independent evolutionary lineages of Btk rif $^{\mathrm{R}}$ passaged as single strain infections (R1K-R4K) or mixed strain infections (R1KT-R4KT). Relative growth rate (RGR) was calculated as: $\log _{\mathrm{e}}$ (final density/initial density). Experiments were conducted on LB plates with $1.5 \%$ agar. Data are means $( \pm \mathrm{SE})$ for each lineage.

Figure 3 The effect of selection regime (mixed strain selection, single strain selection) and presence of a competitor on bacterial productivity (final spore densities) on hard agar plates. Data are the treatment means $( \pm \mathrm{SE})$ for $B t k$ rif $^{\mathrm{R}}$ controls cultured in isolation [Btk control], for total spore production of both strains in competition [Btk $+\mathrm{Btt}$ ], and for each of the competitors in the mixed culture [Btk 
competing with Btt; Btt competing with Btk]. The significance of post hoc treatment contrasts is indicated by: $\dagger P<0.1 ; * P<0.05 ; * * P<0.01$.

Figure 4 The virulence of selected and ancestral $B t k$ rif $^{\mathrm{R}}$ lineages tested in single strain virulence bioassays. The lines are cumulative daily mortality curves for the four strains from the mixed strain (MS) selection treatment (purple lines, R1KT:R4KT); the four strains from the single strain (SS) selection treatment (green lines, R1K:R4K) and their common ancestor in the selection experiment (black symbol, dashed line). Data are the mean daily proportion dying for the $\mathrm{N}=105$ larvae per strain + SE at all three bioassay doses.

Figure 5 The effect of mixed infection with a $B t t \operatorname{spec}^{\mathrm{R}}$ competitor on the virulence of $B t k$ rif $^{\mathrm{R}}$ infections in the ancestral and selected strains. The data are raw daily cumulative mortality for the four lineages from the mixed strain (MS) selection treatment (R1KT:R4KT); the four strains from the single strain (SS) selection treatment (R1K:R4K) and the ancestral Btk rif $^{\mathrm{R}}$ strain (ancestor). In lineage-level analyses (Appendix S1) only one strain (R2K) showed a response to coinfection that was significantly different from the ancestor (hazard coeff $=-1.14$, SE of difference $=$ $0.41, P=0.006)$. All insects were infected with inoculum at 250 spores $\mu \mathrm{l}^{-1}$ of the Btk strain. Open blue circles are the singly-infected controls, filled magenta circles are insects co-infected with both a Btk strain and the ancestral $B t t \operatorname{spec}^{\mathrm{R}}$ strain.

Figure 6 The in vivo growth of $B t k$ rif $^{\mathrm{R}}$ and $B t t \operatorname{spec}^{\mathrm{R}}$ strains from mixed strain (MS) and single strain (SS) selection treatments. (a) The effects of coinfection and selection treatment on the growth of $B t k$ rif $^{\mathrm{R}}$ within hosts, data are means $( \pm \mathrm{SE})$ with $\mathrm{N}=4$ 
independent lineages from each of the selection treatments (single strain selection, mixed strain selection); pairwise comparisons of the effect of coinfection within selection treatment are not significant at the 0.05 level (Tukey's HSD). (b) The relationship between the density of spores from competing $B t t \operatorname{spec}^{\mathrm{R}}$ and $B t k$ rif $^{\mathrm{R}}$ lineages in vivo. Data are log transformed spore counts for cadavers isolated from a single leaf. The plotted line (and closed symbols) represent fitted statistical models for lineages from the single strain selection treatment (SS). The open symbols represent data from the mixed strain selection treatment (MS). 
A

Homogenize pooled cadavers \& plate on selective media

B

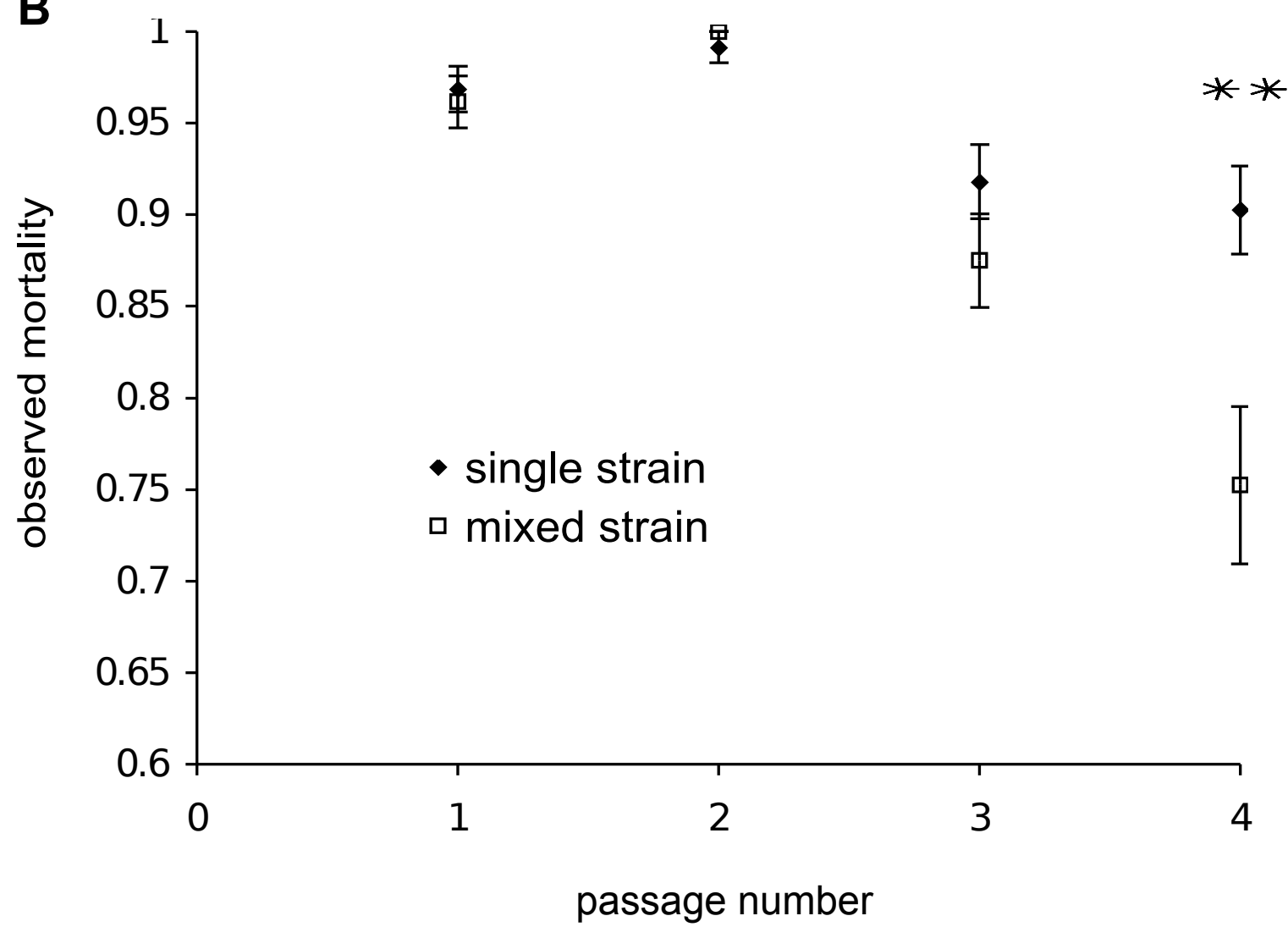

Inoculate 10

leaf discs per

lineage with purified spores
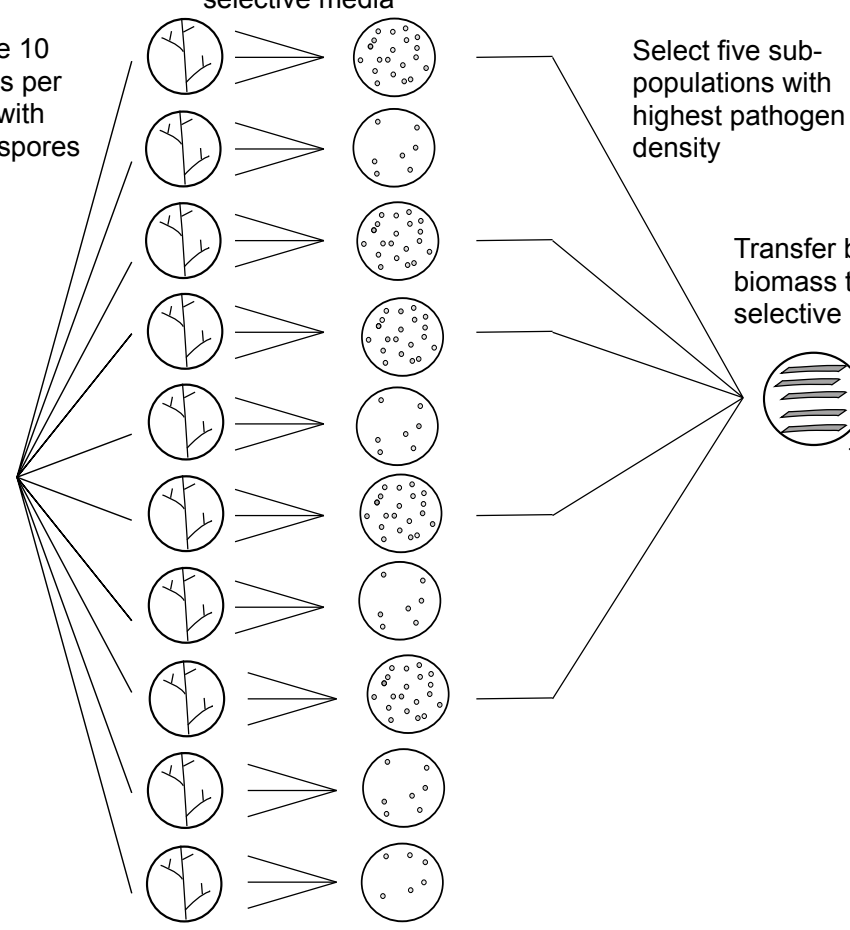

highest pathogen

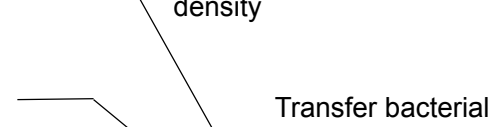

biomass to fresh

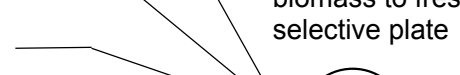

Glycerol stocks and storage at $-80 \operatorname{deg} \mathrm{C}$

Spore production \& purification for next round of infection 



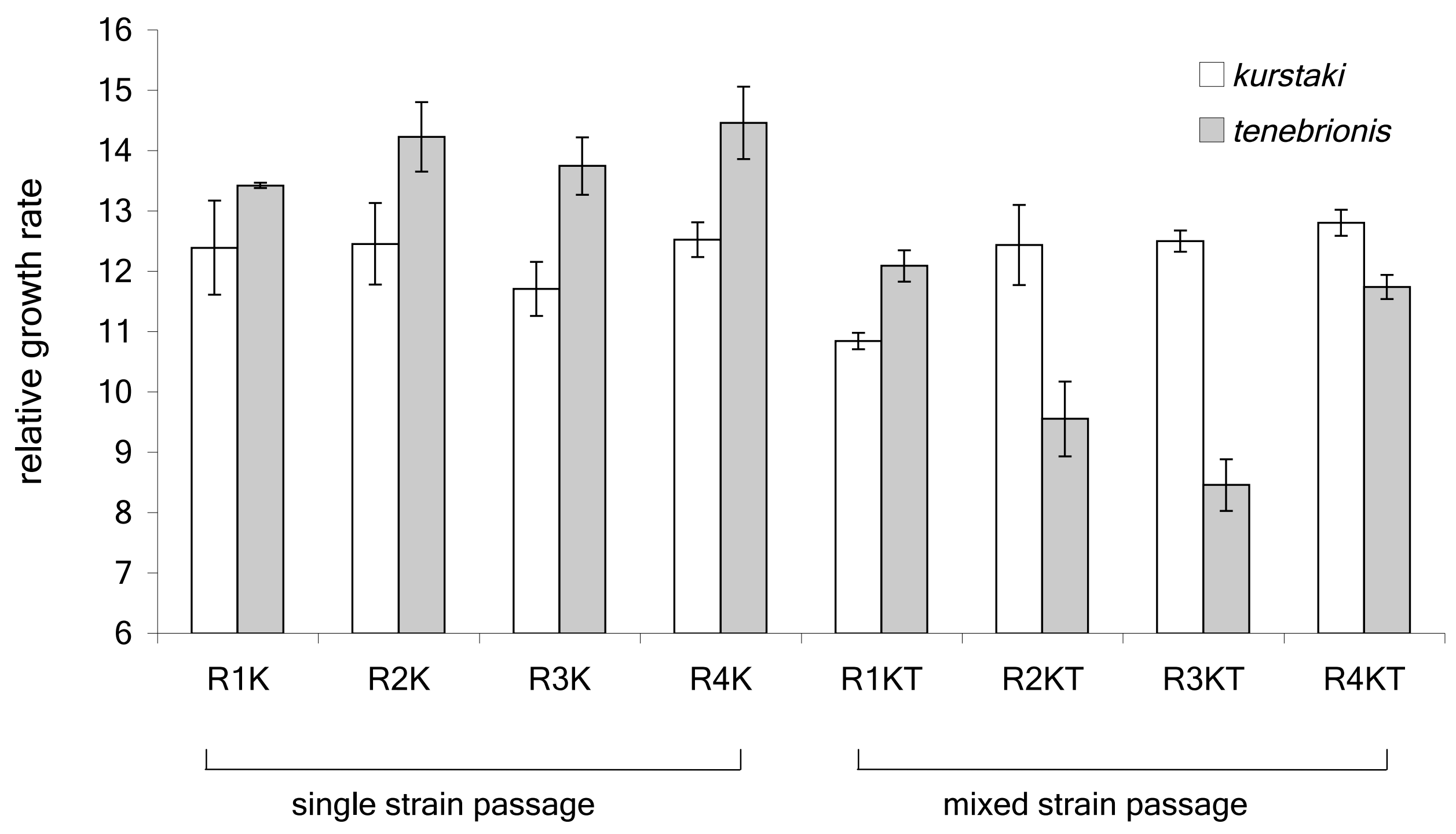




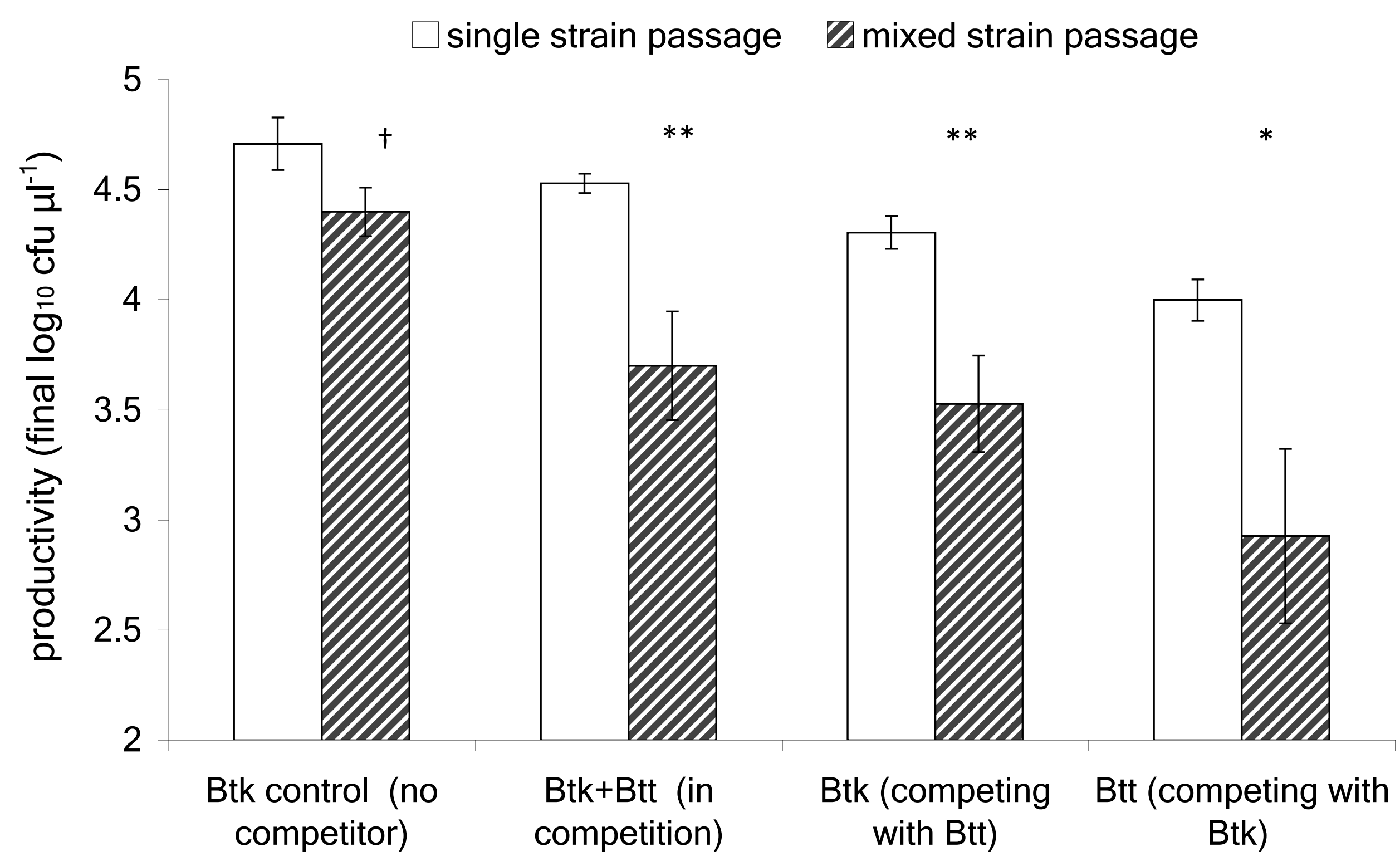




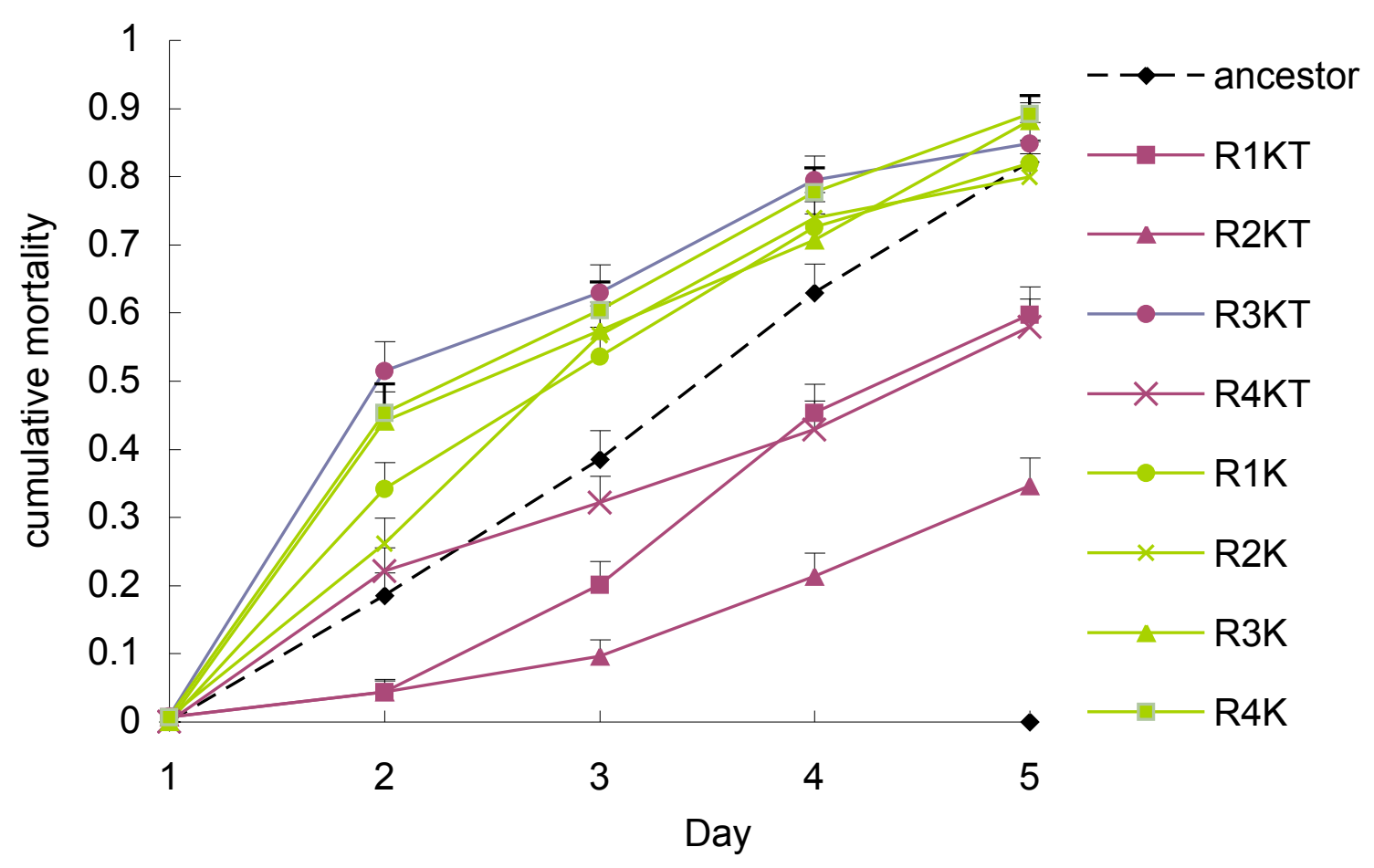




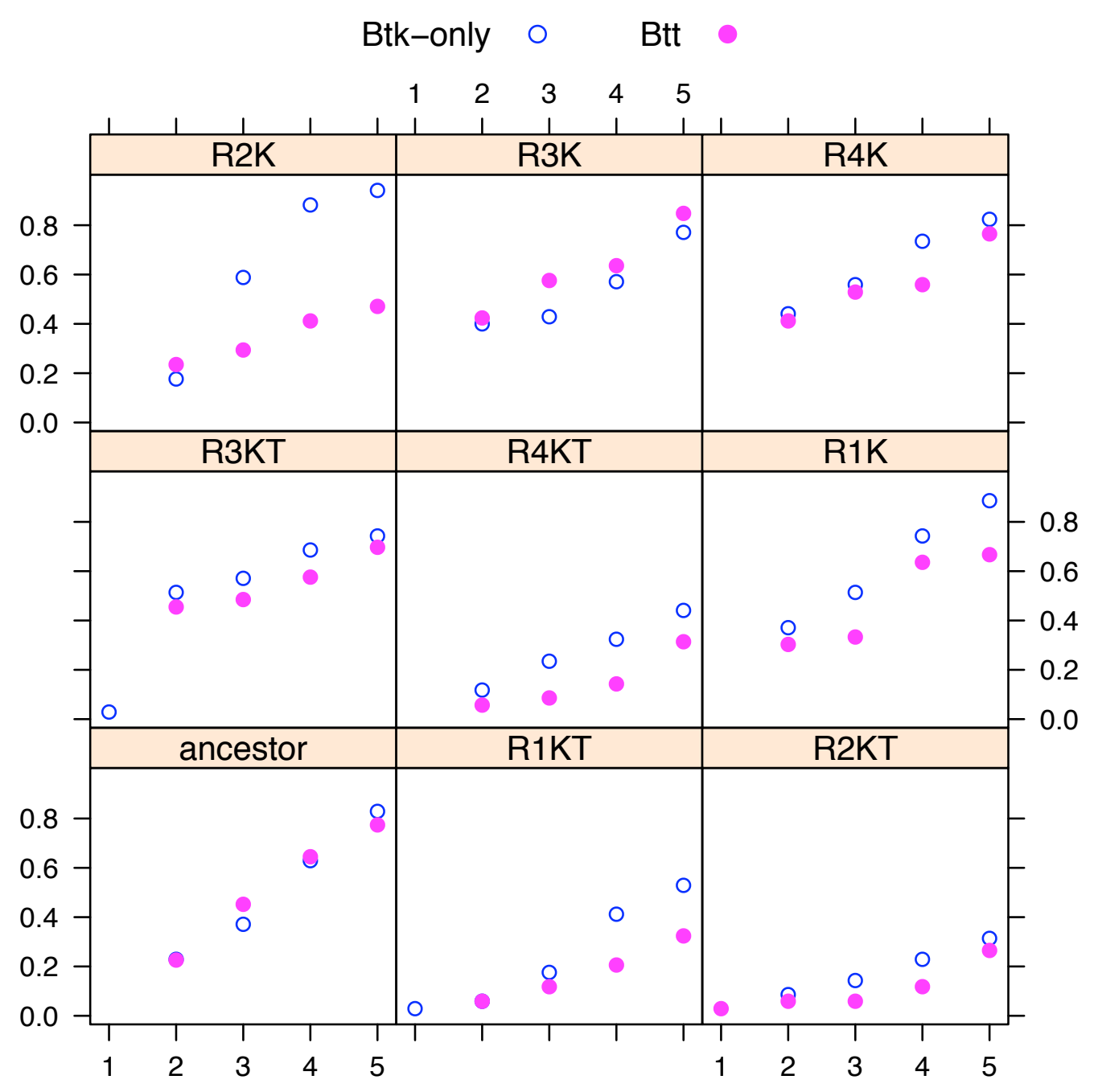



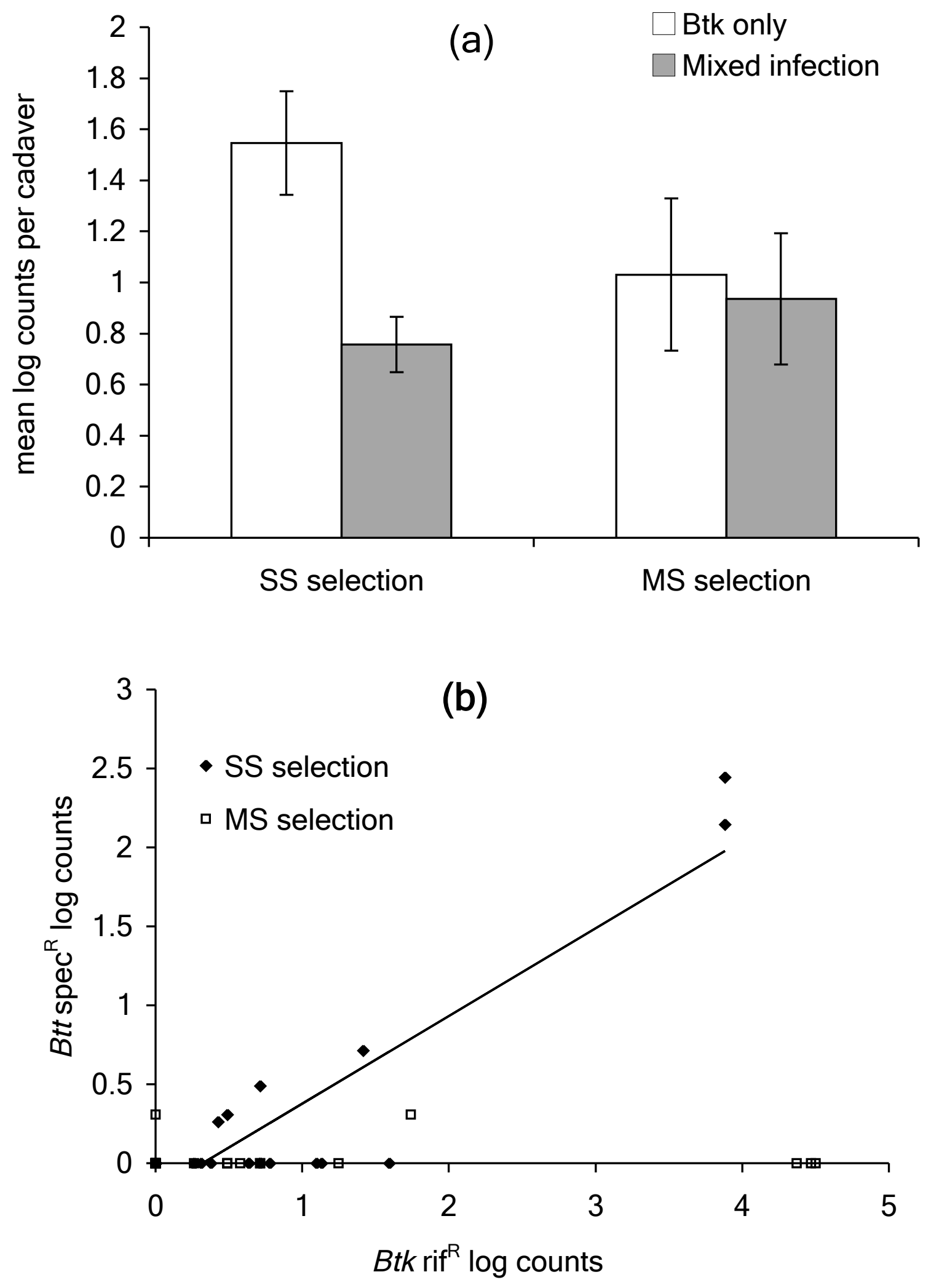